\title{
Mesure de la concentration en gaz d'une émulsion gaz-liquide par absorption de rayons $\gamma$
}

\section{Measurement of the concentration of gas of a gas-liquid emulsion by absorption of $y$ rays.}

\author{
PAR R. HOURS \\ COMMISARIAT A L'ÉNERGIE A'TOMIgUE, \\ SECTION D'APPLICATION DES RADIO-ÉLÉMENTS
}

\begin{abstract}
Par mesure de la "densité optique aux rayons $\gamma$ " d'une émulsion gaz-liquide, on peut atteindre la concentration moyenne en gaz de l'émulsion dans la zone traversée par le faisceau sans perturber le phénomène.

L'energie du rayonnement choisi sera fonction de l'épaisseur d'émulsion à traverser, épaisseur qui, dans le cas de l'eau, ne pourra guère dépasser 1 mètre.

La précision sera proportionnelle da la concentration, à l'épaisseur d'émulsion traversée, au coefficient d'absorption des $\gamma$ dans le liquide, ainsi qu'aux racines carrées de la puissance de la source, de l'efficacité du détecteur et du temps de la mesure. Médiocre aux faibles concentrations, la précision pourra dans certains cas dépasser le 1 pour cent.

L'aspect statistique de la mesure a été étudié. Le mème appareillage pourra servir da toute mesure de densité, notamment celle de toute suspension de particules dans un milieu de densité notablement différente.
\end{abstract}

\section{I. - But de cette étude}

L'objet du présent exposé est de décrire une méthode de mesure de la concentration spatiale en gaz d'un écoulement hétérogène à 2 phases, gaz et liquide, où la phase gazeuse est divisée en un très grand nombre de bulles réparties dans le liquide.

On admettra que l'écoulement est permanent en moyenne dans le temps, et que le fluide est homogène en ce sens que les bulles de gaz sont
By measuring the "optical density for $\gamma$ rays" of a gas-liquid emulsion the average concentration of gas in the zone traversed by the beam of radiation can be obtained. The beam does not perturb the nature of the emulsion. The $\gamma$ energy selected will be a function of the thickness of the emulsion and, for aqueous emulsions, the upper limit of this thickness is about $1 \mathrm{~m}$.

The accuracy will be proportional to the concentration of gas, to the thickness of the emulsion, and to the absorption coefficient of the liquid, and thence to the square roots of the source strength, the detector efficiency, the duration of the measurement. With low concentrations the precision is poor, but in certain cases it can be better than 1 per cent.

The statistical aspects of the measurement have been studied.

The same equipment can be used for density determinations in many other systems, particularly suspensions in which the dispersed phase has a density which is notably different from that of the medium.

petites et distribuées uniformément dans le liquide.

On appelle concentration spatiale en gaz $c$ de l'énuulsion, le volume de gaz contenu dans un volume unité de mélange; (1-c) représente, dans le cas de l'eau, la densité moyenne de l'émulsion.

Nous supposerons que le fluide s'écoule dans une conduite (cas d'une pompe à émulsion ou d'un compresseur hydraulique). 


\section{II. - Principe de la mesure}

Si de part et d'autre de la conduite (fig. 1) nous disposons une source de rayons $\gamma$ et un détecteur de radioactivité, l'activité mesurée par ce détecteur sera fonction de la densité apparente

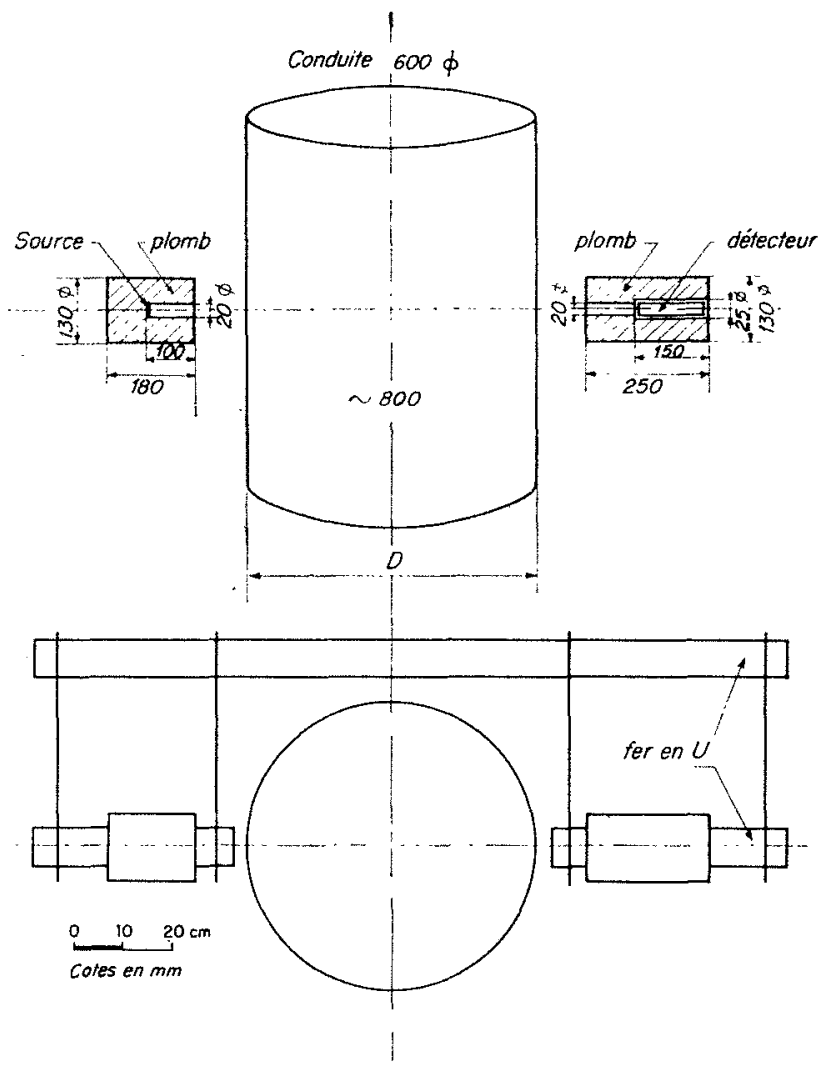

FiG. 1

du fluide traversé par le faisceau, donc de la concentration en gaz $c$ précédemment définie.

De façon plus précise, si l'épaisseur d'émulsion traversée par le faisceau est constante et égale à $D$, les rayons $\gamma$ devront traverser en

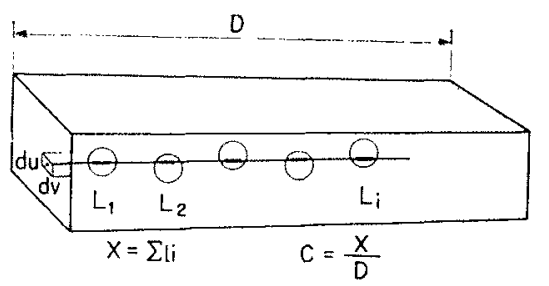

Fig. 2

moyenne, pour atteindre le détecteur, une épaisseur $\mathrm{X}=c \mathrm{D}$ de gaz et une épaisseur $(1-c) \mathrm{D}$ de liquide. Ceci est facile à démontrer : entourons (fig. 2) la droite support d'un photon d'une petite règle de section $d u d v$, de volume $d u d v \mathrm{D}$; on aura:

$$
c=\frac{\text { volume gaz }}{\text { volume émulsion }}=\frac{d u}{d u} \frac{d v}{d v} \frac{\mathrm{X}}{\mathrm{D}}=\frac{\mathrm{X}}{\mathrm{D}}
$$

En intégrant pour tout le faisceau, nous aurons $\bar{c}=(\overline{\mathrm{X}} / \mathrm{D}) \quad(\bar{c}$ et $\overline{\mathrm{X}}$ désignant les valeurs moyennes arithmétiques).

Si le faisceau, limité par des diaphragmes de plomb, est suffisamment étroit pour que le détecteur ne recoive pratiquement pas de photon diffusé, et si la source est monochromatique, nous pourrons écrire :

$$
\begin{aligned}
& \mathrm{I}_{0}=\mathrm{I}_{1} e^{-\mu \mathrm{D}} \\
& \mathrm{I}_{c}=\mathrm{I}_{1} e^{-\mu(1-c) \mathrm{I})}
\end{aligned}
$$

$\mathrm{I}_{o}$ : désignant l'activité, en chocs/seconde par exemple, mesurée par le détecteur pour une concentration $c$;

$\mathrm{I}_{0}$ : activité mesurée pour $c=0$ (liquide pur);

$I_{1}$ : activité mesurée pour $c=1$ (gaz pur);

y. : coefficient d'absorption des photons dans le liquide.

Nous considérons que l'absorption des $\gamma$ dans le gaz est négligeable $\left(u_{\mathrm{gaz}}=0\right)$.

Des équations (1) et (2), nous tirons :

$$
\frac{\mathrm{I}_{\mathrm{s}}}{\mathrm{I}_{0}}=e^{\mu r \mathrm{D}}
$$

d'où :

$$
a=\frac{1}{m_{n} \mathrm{D}} \log \frac{\mathrm{I}_{c}}{\mathrm{I}_{0}}
$$

On voit que $c$ se déduit d'une mesure de «densité optique »; en fait, nous avons affaire à une colorimétrie en très courte longueur d'onde, et retrouverons les mèmes limitations dans la validité de la formule (3) que dans l'emploi de la loi de Beer (voir paragraphe IV).

L'activité $I_{1}$ sera donnée par la formule (4):

$$
\mathrm{I}_{1}=\mathrm{I} \frac{\mathrm{s}}{4 \pi(b+\mathrm{D})^{2}} e^{-\mu_{1} a} \rho
$$

dans laquelle :

$\mathrm{I}=$ activité de la source exprimée en nombre de photons émis par seconde dans tout l'espace;

$s=$ surface 《éclairée 》 du détecteur;

$b=$ (distance source-détecteur) - D;

$a=$ épaisseur totale de parois traversées; 
$\mu_{1}=$ coefficient d'absorption des $\gamma$ dans le matériau de la conduite;

$\checkmark=$ efficacité du détecteur aux $\gamma$ reçus;

(rapport photons détectés/photons reçus).

\section{III. -- Précision de la mesure}

Si l'on traite le calcul de la précision par la méthode élémentaire, on tronve:

$$
\frac{\Delta c}{c}=\frac{\Delta u}{y}+\frac{\Delta \mathrm{D}}{\mathrm{D}}+\frac{\mathrm{I}}{\mu c \mathrm{D}}\left(\frac{\Delta \mathrm{I}_{c}}{\mathrm{I}_{o}}+\frac{\Delta \mathrm{I}_{i}}{\mathrm{I}_{0}}\right)
$$

mais il est plus exact de calculer l'erreur standard (erreur quadratique moyenne) $\sigma_{c}$.

On sait que si : $\mathrm{U}=f(\mathrm{X} Y Z), \mathrm{X}$ Y Z étant des variables indépendantes, nous aurons :

$\sigma_{u}=\sqrt{\left(\frac{\partial U}{\partial X}\right)^{2} \sigma_{X}^{2}+\left(\frac{\partial U}{\partial Y}\right)^{2} \sigma_{Y}^{2}+\left(\frac{\partial U}{\partial Z}\right)^{2} \sigma_{Z}^{2}}$

ce qui nous donne:

$\frac{\sigma_{c}}{c}=\sqrt{\left(\frac{\sigma_{\mu}}{\mu}\right)^{2}+\left(\frac{\sigma_{\mathrm{D}}}{\mathrm{D}}\right)^{2}+\frac{1}{\mu \cdot c \mathrm{D}}\left[\left(\frac{\sigma I_{c}}{\mathrm{I}_{c}}\right)^{2}+\left(\frac{\sigma_{\mathrm{I}_{0}}}{\mathrm{I}_{0}}\right)^{2}\right]}$

Pour le calcul de $\sigma_{I_{c}}$ et $\sigma_{I_{0}}$, nous ne considérerons que les écarts dus à la nature statistique de la désintégration, écarts en général prépondérants.

Nous distinguerons deux cas, suivant que l'on totalise sur une «échelle de comptage » les impulsions individuelles délivrées par le détecteur pendant un temps donne; ou que l'on mesure le courant moyen correspondant grâce à un système intégrateur donnant directement l'activité en chocs/seconde.

\section{$1^{\circ}$ Comptage des impulsions individueldes (MESURE A L'ÉCHELLE).}

Soient $\quad \mathrm{N}_{c}=\mathrm{I}_{e} t_{i}$ et $\mathrm{N}_{0}=\mathrm{I}_{0} t_{0}$ les nombres d'impulsions totalisées pendant les temps $t_{c}$ et $t_{0}$.

Nous savons que $\sigma_{N_{0}}=\sqrt{\mathbf{N}_{t}}$ et $\sigma_{N_{0}}=\sqrt{N_{n}}$, d'où, si l'on néglige les erreurs sur les temps de comptage :

$$
\frac{\sigma \mathrm{I}_{n}}{\mathrm{I}_{o}}=\frac{1}{\sqrt{\mathrm{N}_{s}^{-}}} \text {et } \frac{\sigma \mathrm{I}_{0}}{\mathrm{I}_{0}}=\frac{1}{\sqrt{\mathrm{N}_{0}}}
$$

En portant dans la formule (6), nous obtenons :

$$
\frac{\sigma_{c}}{c}=\sqrt{\left(\frac{\sigma_{\mu}}{\mu}\right)^{2}+\left(\frac{\sigma_{\mathrm{D}}}{\mathrm{D}}\right)^{2}+\frac{1}{\mu^{2} c^{2} \mathrm{D}^{2}}\left[\frac{1}{\mathrm{~N}_{c}}+\frac{1}{\mathrm{~N}_{0}}\right]}
$$

ou, en portant dans la formule (5):

$$
\frac{\Delta c}{c}=\frac{\Delta \mu}{\mu}+\frac{\Delta \mathrm{D}}{\mathrm{D}}+\frac{1}{\mu c \overline{\mathrm{D}}}+\left(\frac{1}{\sqrt{ } \mathrm{N}_{c}}+\frac{1}{\sqrt{\mathrm{N}_{0}}}\right)
$$

Nous discuterons sur la formule (8), moins exacte, mais plus simple que la formule (7).

En ne considérant que le dernier terme de (8), nous voyons que la précision est proportionnelle à $\mu, c, \mathrm{D}, \sqrt{\mathrm{N}_{t}}, \sqrt{\mathrm{N}_{0}}$.

Nous avons trace (fig. 3) des courbes domnant $\sigma_{c} / c$ en fonction de $c$ pour $\mathrm{N}_{c}=\mathrm{N}_{0}=10000 \mathrm{el}$ pour différentes valeur's de $\mu$ et de D (chaque droite correspond à un produit p. D constant pour l'eau). La précision devient médiocre pour de faibles valeurs de $c$.

Toutes choses égales par ailleurs, e'est-àd-dire le produit $\mu c \mathrm{D}$ restant constant ainsi que l'angle solide sous lequel le détecteur est vu depuis la source, la précision est proportionnelle aux racines carrćes de l'activité de la source, de l'efficacité du détecteur [voir formule (4)] et du temps de comptage.

Pour limiter la puissance de la source et le temps du comptage, on aura intérêt à prendre un détecteur de grande efficacité : compteur à scintillation plutôt que compteur Geiger-Müller $\left(^{*}\right)$.

En théorie, on peut, à condition de compter assez longtemps, réduire l'erreur $\sigma_{\mathrm{I}} / \mathrm{I}$ autant que l'on veut; en pratique, on est limité par des questions de durée et d'éventuelle dérive tant de l'appareil de mesure que du phénomène mesuré. II n'en reste pas moins que c'est à l'échelle que l'on aura toujours les mesures les plus précises.

Nous avons jusqu'ici supposé que l'activité mesurée était notablement supérieure au nouvement propre (bruit de fond) du détecteur; s'il n'en était pas ainsi, la précision serait diminuée.

\section{$2^{\circ}$ Mesure du counant Moyen (intégrateur).}

Si le système intégrateur a une constante de temps $\tau$, l'écart standard sur la mesure de $I$ sera :

$$
\frac{\sigma_{\mathrm{I}}}{\mathrm{I}}=\frac{1}{\sqrt{2 \tau \mathrm{I}}}
$$

ce qui conduit à :

$$
\frac{\sigma_{c}}{c}=\sqrt{\left(\frac{\sigma_{\mu}}{\mu}\right)^{2}+\left(\frac{\sigma_{\mathrm{D}}}{\mathrm{D}}\right)^{2}+\frac{1}{2 \tau u^{2} c^{2} \mathrm{D}^{2} \mathrm{I}_{0}}\left(1+\frac{1}{e^{\mu c \mathrm{~b}}}\right)}
$$

(') L'efficacité d'un détecteur varie en général avec l'énergie des photons incidents; pour un compteur G.-M., sa valeur en pour cent est voisine de l'énergie du $\gamma$ exprimée en $\mathrm{MeV}$; pour un cristal scintillant d'INa, elle est de quelques dizaines pour cent, et peut atteindre 100 pour cent pour des $\gamma$ mous. 


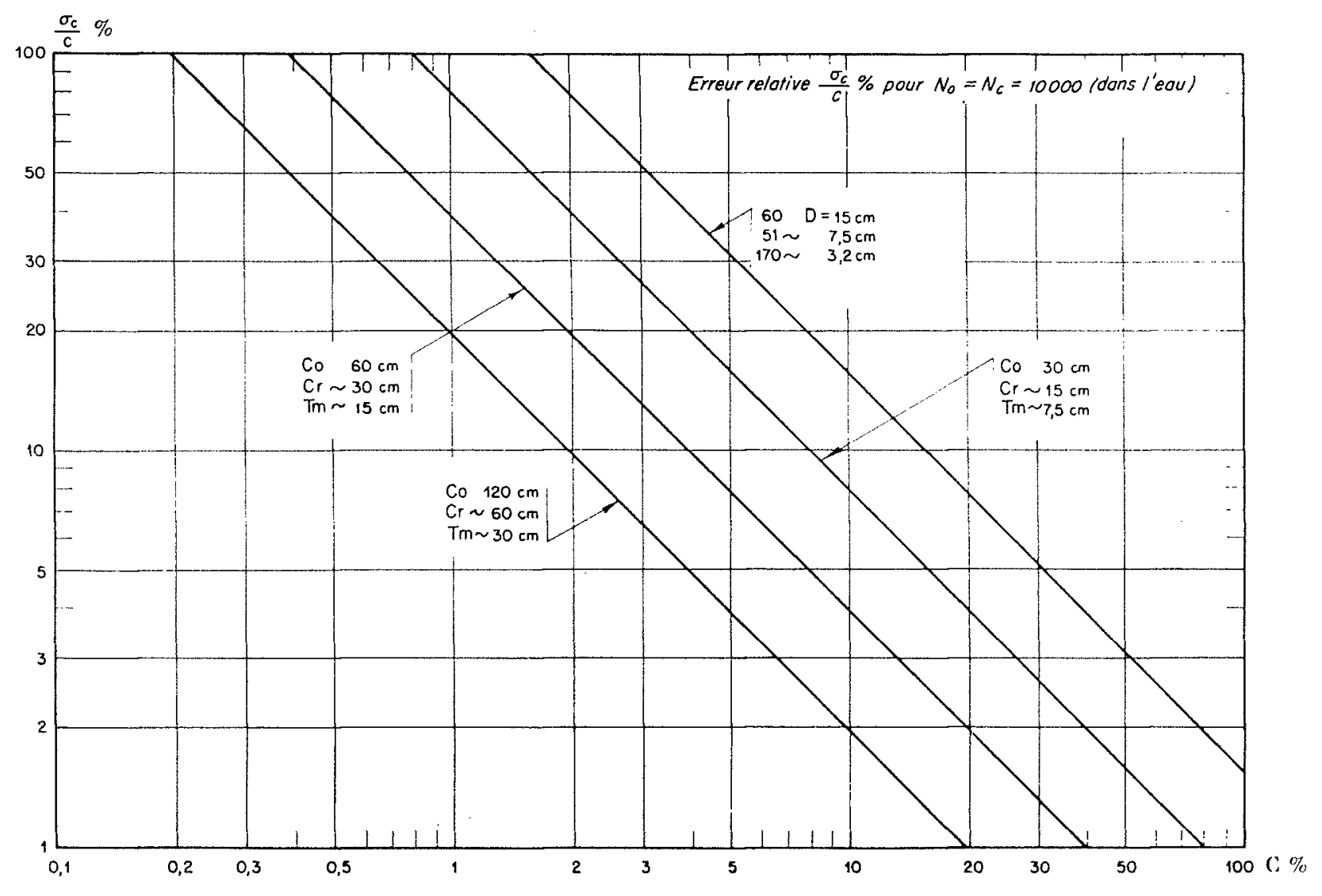

FIG. 3

(10) montre que les erreurs sur $I_{0}$ et $I_{c}$ ne sont plus indépendantes;

(9) montre que l'erreur dans une mesure à l'intégrateur est la même que dans un comptage à l'échelle pendant un temps $2 \tau$. En général, $\tau$ est de l'ordre de quelques secondes, et ne dépasse guère la minute.

Si l'on veut comparer les temps de mesure à l'échelle et à l'intégrateur, il faudra se rappeler qu'avec ce dernier l'aiguille de l'appareil de mesure n'atteint sa valeur finale à $1 \%$ près qu'au bout de $5 \tau$, et à $1 \%$ près qu'au bout de $7 \tau$. De ce fait, il résulte qu'un intégrateur ne pourra pas en général suivre des variations très rapides du phénomène à mesurer ${ }^{\star *}$ ).

La précision de l'intégrateur restera médiocre si l'activité mesurée par le détecteur est faible. Mais, pour une mesure de routine (contrôle continu d'un appareil plutôt qu'étude), l'intégrateur sera préférable à l'échelle, d'autant plus qu'il peut être suivi d'un enregistreur qui compensera en partie sa plus faible précision $\left({ }^{\star \star \star}\right)$ et sur

(**) La mesure à unc précision donnée d'une simple variation d'activité sera cependant moins longue que dans le cas d'une mesure initiale.

$\left({ }^{\star \star}\right)$ Ceci est dû à la possibilité de « lisser 》 la courbe enregistrée. lequel une éventuelle dérive sera plus facile à discerner; rien n'empêche d'ailleurs d'associer une échelle à l'intégrateur.

Nous n'avons pas construit personnellement un ensemble de mesure de caractère industriel, mais de tels systemes sont parfaitement réalisables. Ins utilisent en général comme détecteurs des chambres d'ionisation, nécessitant des sources notablement plus fortes, mais permettant l'emploi d'une chambre de compensation montée en opposition; ce montage augmente notablement la sensibilité et la précision de la mesure; certains constructeurs prétendent ainsi dépasser le pour cent $\left(^{\star \star \star *}\right)$.

\section{IV. - Validité de l'équation (3)}

Il n'est pas indispensable, pour effectuer une bonne mesure, que l'équation (3) soit rigoureusement vérifiée : il suffit en effet d'étalonner l'appareil avec des émulsions de concentration connue; mais il est difficile de réaliser des émul-

(***) P.F. OhMart, H.L. Cook: Applications of radioactive density gaging to process measurement and control. Instrument Society of America Journal, janvier 1955, pp. 16-21. 
sions de concentration parfaitement stable et connue. Comme la mesure de concentration se ramène en définitive à une mesure d'épaisseur de liquide, il sera plus simple, quoique un peu moins rigoureux (voir plus loin parag. 3 et 5) d'étalonner au moyen d'épaisseurs variables de liquide pur.

Il est cependant intéressant d'examiner les différents facteurs limitant la validité de l'équation $(3)$.

\section{Largeur du faisceau :}

L'équation (3) ne suppose pas le faisceau constitué de rayons parallèles, mais seulement la constance de l'épaisseur d'émulsion traversée par chaque photon $\gamma$. Cette condition est pratiquement réalisée si le faisceau est assez mince. On ne peut cependant réduire indéfiniment l'ouverture du faisceau, ce qui conduirait à l'emploi de sources trop puissantes.

Dans le système que nous avons réalisé el utilisé (fig. 1), le faisceau était délimité par d'épais diaphragmes cylindriques de plomb, en sorte que le détecteur était vu depuis la source sous un angle solide de $4 \pi / 40000$ steradians (cercle de $2 \mathrm{~cm}$ de diamètre vu à $1 \mathrm{~m}$ ). La source ctait un disque de cobalt de $20 \mathrm{~mm}$ de diamètre et $2 \mathrm{~mm}$ d'épaisseur, d'une activité de 100 millicuries, donnant 3500 chocs $/ \mathrm{mn}$ à travers $600 \mathrm{~mm}$ d'eau et $12 \mathrm{~mm}$ d'acier (parois du tube) dans un compteur G.-M. de type G1OH. L'alignement de l'ensemble était assuré en faisant glisser les cylindres de plomb sur des fers en U.

Il n'est nullement indispensable d'utiliser un faisceau cylindrique. Ce dernier peut toutefois présenter un avantage dans le cas d'une émulsion figée : par un jeu d'ombre et de pénombre, l'influence sur le détecteur d'une bulle petite vis$\dot{a}$-vis du diamètre du faisceau ne dépendra que dans une faible mesure de la distance de cette bulle à la source.

\section{$2^{\circ}$ Polichromaticité DU RAYONNEMENT DE IA SOURCE :}

On est souvent obligé d'utiliser une source émettant un rayonnement polyénergétique, auquel cas il n'y a plus, à proprement parler, de coefficient d'absorption $u$.

Cependant, si les énergies émises sont voisines, on peut parfois calculer un coefficient $\mu$ moyen suffisamment précis pour la mesure. Dans le cas contraire, il faudra recourir à l'étalonnage. On pourrait tourner la difficulté en utilisant un détecteur délivrant des impulsions d'amplitude proportionnelle à l'énergie des photons reçus (compteur à scintillation), et en effectuant une sćlection d'amplitude; on ne détecterait ainsi que les photons contenus dans une étroite bande d'énergie, pour laquelle il existe un $\mu$ bien défini. Cet artifice est l'équivalent électronique des filtres passe-bande utilisés en colorimétrie.

\section{$3^{\circ}$ POLYChromaticrté DU RAYONNEMENT REÇU paR LE DÉTECTEUR :}

Si le faisceau n'est pas infiniment mince, même en partant d'une source monochromatique, le détecteur recevra toujours une certaine proportion de rayonnement secondaire diffusé par effet Compton avec perte d'énergie. Du fait de cette diffusion, le $\mu$ mesuré par variation de l'épaisseur de liquide traversé sera plus faible que le $\mu$ théorique ou que le $\mu$ donné dans les tables $\left(^{\star}\right)$. Ainsi, dans nos mesures (angle solide $4 \pi / 40000$, épaisseur d'eau $60 \mathrm{~cm}$ ), nous avons trouvé pour le cobalt un $\mu$ de $0,061 \mathrm{~cm}^{-1}$ au lieu de 0,064 donné dans les tables.

OHMART (voir note ${ }^{\star * *}$, p. 639), qui utilise un faisceau très large, trouve un coefficient de $0,026 \mathrm{~cm}^{-1}$.

La figure 3 bis donne une idée de l'importance de la diffusion.

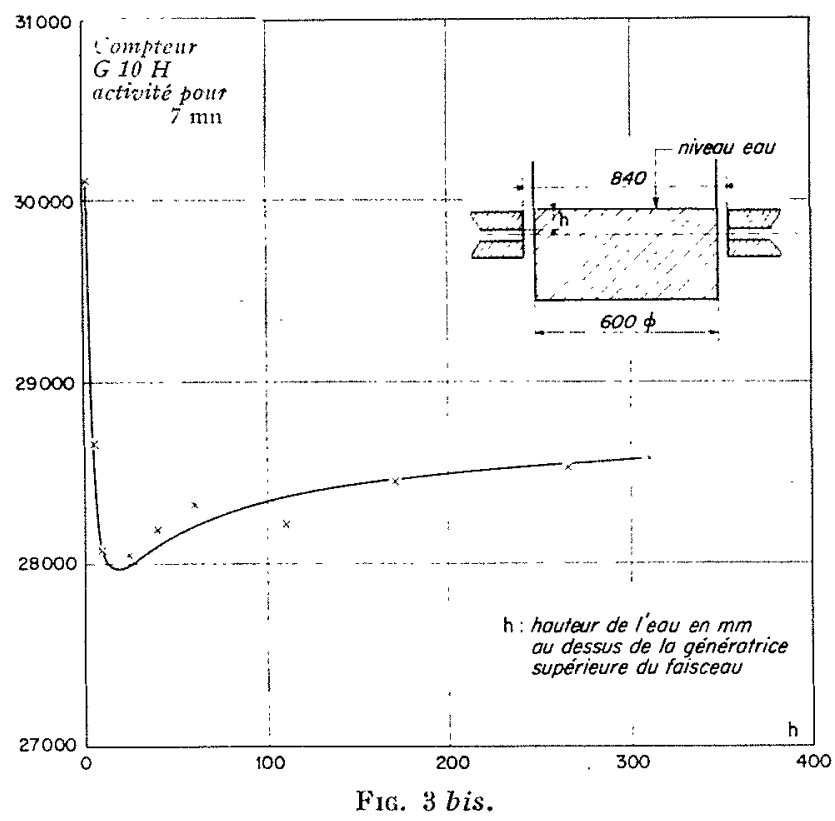

Influence de la diffusion en eau pure.

La diffusion dans une couche de liquide d'épaisseur $c \mathrm{D}$ n'étant pas rigoureusement la même que dans une émulsion de concentration $c$ et d'épaisseur D, l'étalonnage par le liquide pur

(*) Il faut entendre par $\mu$ théorique celvi qui serait mesuré avec un faisceau infiniment mince, tel qu'aucun photon dévié, d'un angle si faible soit-il, ne puisse atteindre le détecteur. 
peut n'être pas absolument valable pour une émulsion dans le cas d'un faisceau large (indépendamment des considérations développées dans le paragraphe 5).

Là encore, on pourra éliminer la difficulté en utilisant un détecteur proportionnel et un sélecteur d'amplitude (voir paragraphe précédent).

\section{$4^{\circ}$ Diffusion SANS PERTE D'ÉNERGIE :}

On pourrait craindre que la traversée des nombreux dioptres gaz-liquide constitués par les bulles introduise une notable diffusion sans perte d'énergie. En réalité, cette diffusion, tout à fait négligeable pour des photons de quelques centaines de $\mathrm{keV}$, restera extrêmement faible même pour les photons les plus mous utilisés.

L'indice de réfraction air-eau est :

$$
n=1-3,6 \cdot 10^{-8}
$$

pour des photons de $80 \mathrm{keV}$,

$$
n=1-3,6 \cdot 10^{-6}
$$

pour des photons de $8 \mathrm{keV}$; pour ces derniers, l'ouverture d'un faisceau incident parallèle est, après traversée d'une émulsion, inférieure à $10^{-4}$ radian.

\section{5" Erreur systématique due a la Nature de L'ÉMULSION :}

Cette erreur est due à la fois à la nature même de l'émulsion et à l'absorption exponentielle du rayonnement dans le liquide.

Si un photon doit, pour atteindre le détecteur, traverser une épaisseur $(\mathrm{D}-\mathrm{X})$ de liquide, sa probabilité d'atteindre le détecteur est $e^{-\mu(\mathrm{D}-\mathrm{X})}$, $X$, trajet du photon dans le gaz, étant une variable aléatoire de valeur moyenne $\overline{\mathrm{X}}=c \mathrm{D}$.

$\mathrm{Si}$, pendant le temps $t$ de la mesure, la source a émis $\mathrm{N}=\mathrm{I} t$ photons dans la direction $d u$ détecteur, celui-ci n'en recevra en moyenne que:

$$
\overline{\mathrm{N}}_{\mathrm{X}}=\mathrm{I} t \overline{\mathrm{e}^{-\mu(\mathrm{D}-\mathrm{X})}}=\mathrm{I}_{0} t \overline{e^{\mu \mathrm{x}}} \neq \mathrm{I}_{0} t e^{\overline{\mu \mathrm{x}}}
$$

Or, c'est $\overline{N_{X}}$ que nous mesurons, mais $\bar{X}$ que nous voulons atteindre. La valeur $X$ calculée par la formule (3), que nous désignerons par $\mathrm{X}_{\mathrm{cal}}$, sera donc différente du $\overline{\mathrm{X}}$ cherché.

$$
\mathrm{X}_{\text {ral. }}=\frac{1}{u}\left(\log \bar{I}_{X}-\log I_{n}\right)
$$

tandis que :

$$
\overline{\mathrm{X}}=\frac{1}{\mathrm{t} .}\left(\log \mathrm{I}_{\overline{\mathrm{x}}}-\log \mathrm{I}_{0}\right)
$$

Pour calculer $I_{x}$; nous utiliserons la formule suivante :

$$
\overline{f(x)}=f(\bar{x})+\frac{\overline{(x-\bar{x})}}{1 !} f^{\prime}(\bar{x})+\frac{\overline{(x-\bar{x})^{2}}}{2 !} f^{\prime \prime}(\bar{x})+\ldots
$$

Le terme $\overline{(x-\bar{x})}$ est nul par définition.

Dans notre cas, $f(x)=e^{\mu \mathrm{x}}$, d'où :

$$
\overline{e^{\mu \mathrm{X}}}=e^{\mu \mathrm{X}}\left[1+\frac{\overline{(\mathrm{X}-\overline{\mathrm{X}})^{2}}}{2 !} \mu^{2}+\frac{\overline{(\mathrm{X}-\overline{\mathrm{X}})^{3}}}{3 !} \mu^{3}+\ldots\right]
$$

Il nous faut calculer $\overline{(\mathrm{X}-\overline{\mathrm{X}})^{2}}=\mathrm{r}_{\mathrm{X}}^{2}$, variance, ainsi que les « variances » d'ordre supérieur. Pour cela, nous ferons plusieurs hypothèses simplificatrices : nous supposerons les bulles sphériques et égales, de diamètre $d$, les rayons parallèles, la source carrée de côté $a$.

Si $V$ est la vitesse d'écoulement de l'émulsion dans la conduite, il aura défilé pendant le temps $t$ de la mesure un volume $a \mathrm{~V} t \mathrm{D}$ d'émulsion, contenu dans un parallélépipède rectangle (fig. 4)

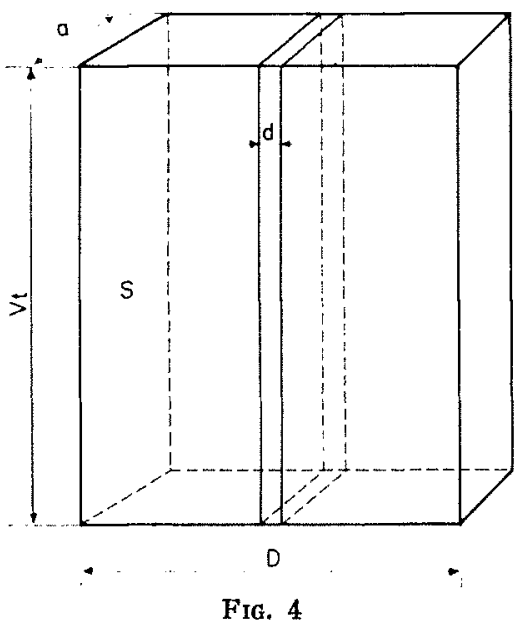

dont une face peut être assimilée à une source plane rectangulaire de surface $\mathrm{S}=\alpha \mathrm{V} t$ qui aura émis I $t$ photons dans une direction normale à la source. Ce volume contient $6 c \mathrm{~S} \mathrm{D} / \pi d^{3}$ bulles.

Pour calculer $\overline{(\mathrm{X}-\overline{\mathrm{X}})^{2}}$, nous considérerons que les bulles sont réparties dans $\mathrm{D} / d$ tranches verticales d'épaisseur $d$ contenant chacune $n=6 c \mathrm{~S} / \pi d^{2}$ bulles; cette hypothise, admissible tant que les bulles ne sont pas trop serrées, permet d'éliminer certaines difficultés de calcul dues au fait que les bulles ne peuvent s'interpénétrer (empêchements stériques).

La probabilité qu'un photon iraversant une tranche y rencontre une bulle est :

$$
p=\frac{n \pi d^{2}}{4 \mathrm{~S}}=\frac{3 c}{2}
$$


La probabilité que cette bulle déjà rencontrée le soit sur une longueur comprise entre $l$ et $l+d l$ est (fig. 5) :

$$
d p=\frac{2 \pi \rho d}{\pi d^{2} / 4}=\frac{2 l d l}{d^{2}}
$$

ce qui donne:

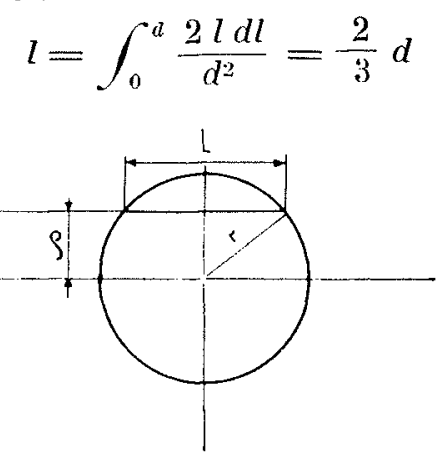

FIG. 5

La longueur moyenne $\bar{x}$ parcourue dans le gaz dans la traversée d'une tranche sera :

$$
\bar{x}=p \bar{l}=\frac{2 d}{3} \cdot \frac{3 c}{2}=c d
$$

Calculons :

$$
\begin{gathered}
\tau_{x}^{2}=\overline{(x-\overline{\bar{x}})^{2}}=\overline{x^{2}}-\bar{x}^{2} \\
\overline{x^{2}}=\int_{0}^{l l} l^{2} \cdot \frac{3 c}{2} \cdot \frac{2 l d l}{d^{2}}=\frac{3}{4} c d^{2}
\end{gathered}
$$

d'où :

$$
\sigma_{\mathrm{x}}^{2}=c d^{2}\left(\frac{3}{4}-c\right)
$$

Dans la traversée de $\mathrm{D} / d$ tranches, le parcours moyen $\overline{\mathrm{X}}$ du photon dans le gaz sera:

$$
\overline{\mathrm{X}}=\overline{\mathrm{x}} \stackrel{\mathrm{D}}{\mathrm{d}}=c \mathrm{D}
$$

La variance de $\mathrm{X}$ sera la somme des variances $\sigma_{x}^{2}$ pour chaque tranche $\left(^{*}\right)$ :

$$
\sigma_{\mathrm{X}}^{2}=\frac{\mathrm{D}}{d} \sigma_{x}^{2}=c d \mathrm{D}(3 / 4-c)
$$

On calculerait de même :

$$
\overline{(\mathrm{X}-\overline{\mathrm{X}})^{3}}=\overline{\mathrm{X}^{3}}-3 \overline{\mathrm{X}^{2}} \overline{\mathrm{X}}+2 \overline{\mathrm{X}}^{3}
$$

le calcul donne :

$$
(\overline{\mathrm{X}-\mathrm{X}})^{5}=c d^{2} \mathrm{D}\left(3 / \mathrm{S}-9 / 4 c+2 c^{2}\right)
$$

(*) Nous n'avons pas tenu compte des fluctuations de la concentration dans chaque tranche; elles seront en général faibles, vu le grand nombre de bulles mises en jeu, et n'interviendront qu'au second ordre.
Nous ne développerons pas la suite des calculs; le terme du troisième ordre, dans l'équation (12), est en général déjà négligeable. En ne conservant que le terme en $\sigma_{\mathrm{x}}{ }^{2}$, l'équation (12) nous donne :

$$
\mathrm{I}_{\mathrm{x}}=\mathrm{I}_{0} e^{\mu \mathrm{x}}\left(1+\frac{\mu^{2}}{2} \sigma^{2} \underline{x}\right),
$$

d'où d'après (3) :

$$
\begin{aligned}
& \mathrm{X}_{\mathrm{cal} .}=\frac{1}{\mu} \log e^{-\mu \mathrm{x}}\left(1+\frac{\mu^{2}}{2} \sigma_{\mathrm{x}}^{2}\right) \\
& \mathrm{X}_{\mathrm{cal} .}=\overline{\mathrm{X}}+\frac{1}{\mu} \log \left[1+\frac{\mu \cdot=}{2} c d \mathrm{D}(3 / 4-c)\right]
\end{aligned}
$$

Souvent, $\left(\mu^{2} / 2\right) c d \mathrm{D}(3 / 4-c)<<1$, et l'on pourra écrire la formule simplifiée :

$$
\mathrm{X}_{\mathrm{eal.}}=\overline{\mathrm{X}}+\frac{\mu c d \mathrm{D}}{2}(3 / 4-c)
$$

d'où :

$$
\frac{\mathrm{C}_{\mathrm{cal} .}-\overline{\mathrm{C}}}{\mathrm{C}}=\frac{\mu d}{2}(3 / 4-c)=\frac{d}{2 \lambda}(3 / 4-c)
$$

La concentration calculée par la formule (3) sera donc systématiquement trop forte; mais l'erreur ainsi commise sera négligeable tant que le diamètre des bulles restera petit devant le libre parcours moyen $\lambda=(1 / \mu)$ des photons dans le liquide.

\section{Extmple:}

Cobalt et eau : $\mu=0,064, \mathrm{D}=60 \mathrm{~cm}, c=25 \%$, $d=0,06 \mathrm{~cm}$, conditions correspondant à nos mesures.

$\left(\mu^{2} / 2\right) c d \mathrm{D}(3 / 4-c)=0,009$, on peut donc utiliser la forme simplifiée (16), qui donne $(\Delta c / c) \# 1 \%$.

Dans certains cas cependant (rayonnement très mou ou liquide très dense), cette correction ne sera plus négligeable.

L'intérêt de ce calcul vient de ce que la correction devra être appliquée même dans le cas où l'appareillage aura été étalonné avec des épaisseurs variables de liquide pur.

Si la concentration devenait si grande que les bulles forment une sorte de réseau rhomboédrique régulier, la méthode du découpage en tranches d'épaisseur $d$ ne serait plus valable, pas plus que la simple addition des variances (variables non indépendantes); la correction serait alors plus importante que celle donnée par l'équation (14), mais nous ne l'avons pas calculée. 


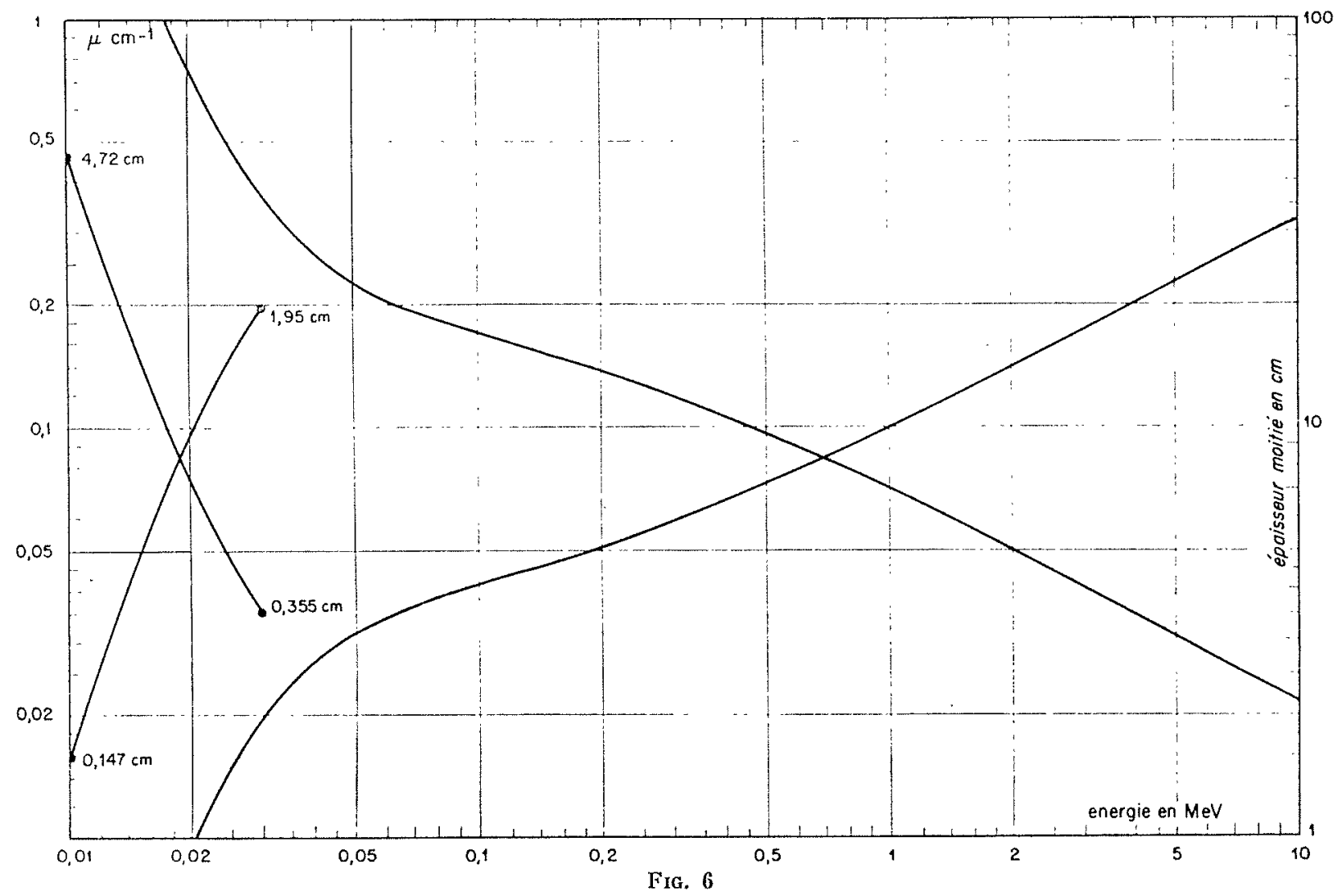

Coefficient d'absorption des $\gamma$ dans l'eau, $\mu$, en $\mathrm{cm}^{-1}$

(Valeurs extraites de Siegbahn : " $\beta$ and $\gamma$ ray spectroscopy", p 873).

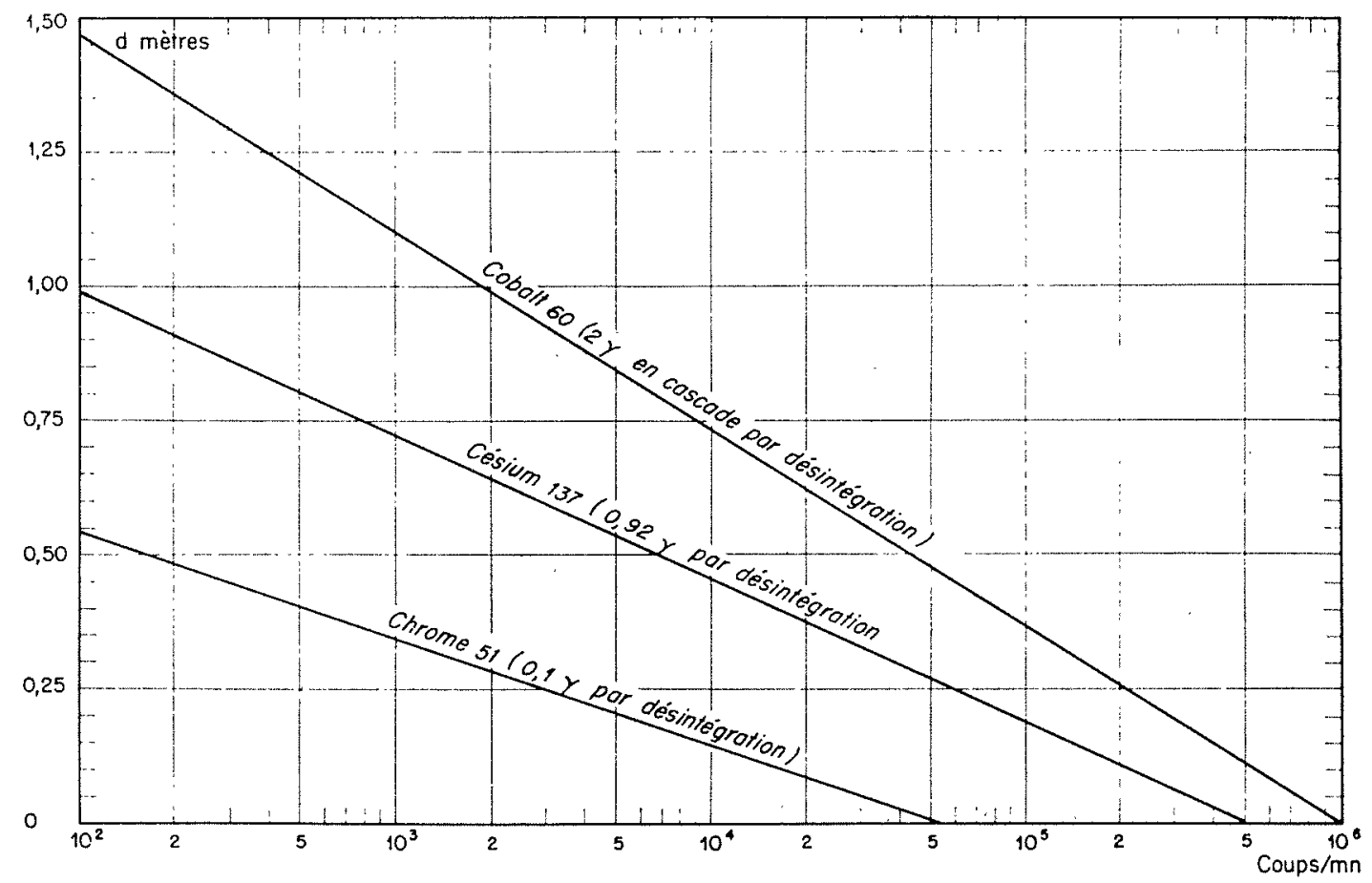

FiG. 7

Activité en coups/mn délivrée par un détecteur d'efficacité $=1$ pour cent pour une source de 1 curie vue à travers une épaisseur $d$ (mètres) d'eau sous un angle solide $4 \pi / 40.000$ steradians correspondant à un cercle de $2 \mathrm{~cm}$ de diamètre vu à 1 mètre (l'absorption de la paroi du tube a été négligée). 


\section{V. - Choix des sources radioactives}

Nous avons vu que la précision est proportionnelle à $y$. Cependant, la puissance des sources étant limitée par des considérations de production autant que de protection, on ne pourra pas toujours utiliser un $\mu$ très grand.

La figure 6 donne les $n$ (et les épaisseurs moi- tié correspondantes) pour l'eau pour des énergies de $10 \mathrm{keV}$ à $10 \mathrm{MeV}$.

La figure 7 donne l'activité mesurée dans un détecteur d'efficacité $?=1 \%$, vu sous un angle solide de $4 \pi / 40000$ steradians pour différentes sources de 1 curie.

Voici quelques émetteurs utilisables avec leur $\mu$ et leur épaisseur moitié dans l'eau :

\begin{tabular}{|c|c|c|c|c|}
\hline Emetreun & RAYONNEMENT & PÉriode & $\mu \mathrm{cm}^{-1}$ & $e_{172} \mathrm{~cm}$ \\
\hline $60_{\mathrm{C}}$ & $(1,17-1,33 \mathrm{MeV}$ en cascade $)$ & 5,3 ans & 0,064 & 10,8 \\
\hline $137_{\mathrm{ss}}$ & $(92 \%$ de $0,66 \mathrm{MeV})$ & 33 ans & 0,0862 & 8,4 \\
\hline $51_{\mathrm{rr}}$ & $(10 \%$ de $0,325 \mathrm{MeV})$ & 26 jours & 0,117 & 5,9 \\
\hline $170_{\mathrm{Tm}}$ & $(\mathrm{qq} \% \gamma 84 \mathrm{keV}+\mathrm{X} 52 \mathrm{keV})$ & 129 jours & $\sim 0,221$ & $\sim 3,13$ \\
\hline $192_{\mathrm{Tr}}$ & (nombreux $\gamma$ de 0,3 à $0,7 \mathrm{MeV}$ ) & 70 jours & & \\
\hline
\end{tabular}

Pour des rayonnements plus mous, il existe quelques autres isotopes possibles. ${ }^{155} \mathrm{Eu},{ }^{188} \mathrm{~W}$, ${ }^{14}{ }^{4} \mathrm{Pm},{ }^{20 \pm} \mathrm{Tl}$ ), intéressants surtout par leurs X caractéristiques et leur rayonnement de freinage interne; enfin, des émetteurs $\beta$ ( ${ }^{90} \mathrm{Sr}$, période 25 ans, $\beta$ de 2 Mev, par exemple), irradiant des cibles convenablement choisies, constituent de petits émetteurs de rayons $\mathrm{X}$ fort utiles dans ce genre de problème.

La plupart de ces isotopes n'émettent pas un rayonnement monoénergétique, mais nous avons vu que ceci ne constitue pas un inconvénient majeur.

\section{VI. - Réalisation et essais}

L'appareil décrit au paragraphe IV-1 a été utilisé pour des mesures sur le diamètre et différentes cordes d'une canalisation de $60 \mathrm{~cm}$ de diamètre : les résultats trouvés ont été en parfait accord avec des mesures pneumatiques simultanées.

\section{VII. - Généralisation de la méthode}

Cette méthode se généralise immédiatement au cas d'une suspension de particules de coefficient d'absorption $\mu^{\prime}$ dans un liquide de coefficient $\mu$; il suffit de remplacer $\mu$ par $\left(\mu-\mu^{\prime}\right)$.
La formule (3) devient :

$$
c={\frac{1}{\left(\mu-\mu^{\prime}\right)} \overline{\mathrm{D}}}^{\frac{\log \mathrm{I}_{0}}{\log \mathrm{I}_{0}}}
$$

et la formule (16) devient :

$$
\frac{\Delta c}{c}=\frac{\left(\mu-u^{\prime}\right) d}{2} \cdot(3 / 4-c)
$$

la différence systématique $\bar{c}-c_{\text {calcule }}$ étant $\mathrm{du}$ signe de $\left(\mu^{\prime}-\mu\right)$.

Ainsi, dans le cas de sable en suspension dans l'eau et d'une source de cobalt :

$$
\mu_{\text {eau }}=0,064 \quad \mu_{\text {sable }}^{\prime}=0,115
$$

$\mu^{\prime}-\mu=0,051$

on aura des résultats voisins de ceux obtenus pour l'air dans l'eau, mais en sens inverse ( $I_{c}$ diminuant quand $c$ croît).

La mesure sera d'autant plus précise que la différence $\left(\mu-\mu^{\prime}\right)$ sera grande, c'est-à-dire que les deux corps en présence auront des densités différentes en valeur absolue.

Nous tenons à remercier, en terminant, tous ceux qui ont participé à cette étude : MM. MARQUENET et KAMPÉ DE FERRIET, Ingénieurs à l'Electricité de France, M. P. LÉvềue, Chef de la S.A.R. au C.E.A., ainsi que M. Guy Maxer, du Service de Chimie Physique au C.F.A., qui nous a conseillé pour la solution de certaines difficultés d'ordre mathématique. 
D I S C U S I I N

Président: M. Laval

M. Larras remarque que la concentration est mesurée suivant un diamètre horizontal, mais qu'elle varie suivant la hauteur, d'après la communication précédente.

M. Houns répond que la mesure est faite suivant un diamètre horizontal et que, de toute facon, il s'agit d'un laisceau étroit $(2 \mathrm{~cm})$. On pourrait, d'ailleurs, utiliser un détecteur avec un faisceau plus mince $(1 \mathrm{~mm})$ qui présenterait beaucoup d'avantages da point de vue de la diffusion, mais seulement sur une épaisseur d'émulsion assez réduite pour éviter l'emploi d'une source trop puissante.

M. Larras signale qu'il se produit des rayomnements dans la conduite, notamment au contact du liquide avec les bulles ou la paroi et que ces rayonnements sont la cause de pertes d'énergie non prises en compte dans les formules; il y a toujours, en effet, lorsqu'on change de milieu, une partie du rayonnement qui est réfléchi sur la paroi et cela peut modifier notamment le coefficient. pourcentage de bulles de la formule en fonction des dimensions des bulles.

M. Harbrons interprète ce phénomène de la façon suivante : lorsqu'il y a diffusion d'un photon, il se produit une nouvelle diffusion du faisceau originaire; il n'a pas l'impression qu'il en soit tenu compte dans les conditions de ces recherches.
M. Hours répond que M. Larras et M. Halbrone font allusion au problème de la diffusion sans perte d'énergie, mais qu'à son avis, ce phénomène ne doit pas avoir une inlluence notable sur les mesures, car, vu les longueurs d'onde utilisées, les indices de réfractions sont très voisins de l'unité. Cet aspect de la question sera précisé dans le texte définitif de la publication.

M. Halbrone signale une méthode d'étalonnage des sondes électriques, consistant à faire osciller à vitesse modérée, autour d'un axe horizontal, un cube a parois transparentes, contenant une certaine hauteul d'eau au repos; il se crée une émulsion homogène, dont la concentration en air est donnée par la valeur du rapport: hauteur d'air au repos/arète du cube.

'M. Houns indique qu'il peut être intéressant d'employer un mélange d'air et de paraffine car, en refroidissant, on peut étudier la concentration des bulles et voir dans quelle mesure la correction systématique est valable; néanmoins, il est difficile d'obtenir par ce procédé une émulsion homogène.

Sur une remarque de $M$. Serpatud, relative aux matières solides en suspension dans l'eau, M. Houns indique qu'il faudrait considérer la différence des coefficients d'absorption $\left(\mu_{1}-\mu_{2}\right)$. Le fait d'utiliser une suspension de baryte à coefficient d'absorption élevé serait beaucoup plus favorable.

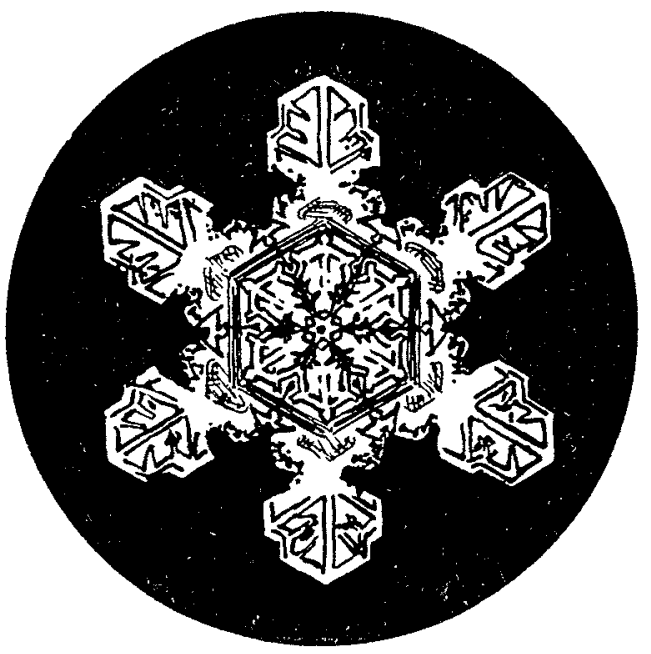

